\title{
Discrete operators associated with linking operators
}

\author{
Margareta Heilmann ${ }^{1}\left[\right.$ [ $\cdot$ Fadel Nasaireh ${ }^{2} \cdot$ Ioan Raşa $^{2}$ \\ Received: 29 September 2018 / Revised: 23 October 2020 / Accepted: 26 October 2020 / \\ Published online: 4 November 2020 \\ (c) The Author(s) 2020
}

\begin{abstract}
We associate to an integral operator a discrete one which is conceptually simpler, and study the relations between them. The differences between the integral and the associated discrete operators are estimated. Applications are given, involving the Baskakov type operators, genuine Baskakov-Durrmeyer type operators, and their Kantorovich modifications. We construct the discrete operators associated with these integral operators and apply our general results in this context.
\end{abstract}

Keywords Linking operators · Baskakov-Durrmeyer type operators · Kantorovich modifications of operators

Mathematics Subject Classification 41A36 · 41A35 · 41A28

\section{Introduction and notation}

It is well-known that integral operators are very useful in Approximation Theory. However, sometimes they are expressed in terms of complicated integrals with respect to complicated measures. In this paper we associate to an integral operator a discrete one which is conceptually simpler, and study the relations between them. Some results in this direction can be found also in [13].

$\bowtie \quad$ Margareta Heilmann

heilmann@math.uni-wuppertal.de

Fadel Nasaireh

fadelnasierh@gmail.com

Ioan Raşa

Ioan.Rasa@math.utcluj.ro

1 School of Mathematics and Natural Sciences, University of Wuppertal, Gaußstraße 20, 42119 Wuppertal, Germany

2 Department of Mathematics, Technical University, Str. Memorandumului 28, 400114 Cluj-Napoca, Romania 
After introducing the necessary definitions we present some general results. Then we recall the definitions of Baskakov type operators, genuine Baskakov-Durrmeyer type operators, and their Kantorovich modifications. We construct the discrete operators associated with these integral operators and apply our general results in this context.

\section{Preliminaries}

In what follows the monomials $e_{j}, j \in \mathbb{N}_{0}$, are given by $e_{j}(x)=x^{j}$.

Let $I \subset \mathbb{R}$ be an interval and $H$ a subspace of $C(I)$ containing $e_{0}, e_{1}$ and $e_{2}$. Let $L: H \longrightarrow C(I)$ be a positive linear operator such that $L e_{0}=e_{0}$. The second moment of $L$ is defined by

$$
M_{2} L(x)=L\left(e_{1}-x e_{0}\right)^{2}(x), x \in I
$$

For a fixed $x \in I$ consider the functional $H \ni f \longrightarrow L f(x)$ and define $\operatorname{Var}_{x} L:=$ $L e_{2}(x)-\left(L e_{1}(x)\right)^{2}$; then, roughly speaking, $\operatorname{Var}_{x} L$ shows how far is the functional from being a pointwise evaluation.

It is easy to verify that

$$
M_{2} L(x)-\operatorname{Var}_{x} L=\left(L e_{1}(x)-x\right)^{2} .
$$

Now let $L$ be of the form

$$
L f:=\sum_{j=0}^{\infty} A_{j}(f) p_{j}, f \in H
$$

where $A_{j}: H \longrightarrow \mathbb{R}$ are positive linear functionals,

$$
A_{j}\left(e_{0}\right)=1 \text { and } p_{j} \in C(I), p_{j} \geq 0, \sum_{j=0}^{\infty} p_{j}=e_{0}
$$

Let

$$
b_{j}:=A_{j}\left(e_{1}\right), \operatorname{Var} A_{j}:=A_{j}\left(e_{2}\right)-b_{j}^{2}, j \geq 0
$$

Then, generally speaking, $\operatorname{Var} A_{j}$ shows how far is $A_{j}$ from the point evaluation at $b_{j}$.

The discrete operator associated with $L$ is defined by

$$
D: H \longrightarrow C(I), D f:=\sum_{j=0}^{\infty} f\left(b_{j}\right) p_{j}
$$

The point evaluation functional at $b_{j}$ is conceptually simpler than $A_{j}$; from this point of view, $D$ is simpler than $L$. We shall investigate the relations between $L$ and $D$. 
It is easy to verify that

$$
M_{2} D(x)=\sum_{j=0}^{\infty}\left(b_{j}-x\right)^{2} p_{j}(x), x \in I .
$$

Moreover, according to (1) and (3)

$$
\begin{aligned}
M_{2} L(x)-\operatorname{Var}_{x} L & =\left(\operatorname{Le}_{1}(x)-x\right)^{2} \\
& =\left(\sum_{j=0}^{\infty}\left(b_{j}-x\right) p_{j}(x)\right)^{2} \\
& =\left(\sum_{j=0}^{\infty}\left(b_{j}-x\right) \sqrt{p_{j}(x)} \sqrt{p_{j}(x)}\right)^{2} \\
& \leq \sum_{j=0}^{\infty}\left(b_{j}-x\right)^{2} p_{j}(x) .
\end{aligned}
$$

Combined with (6), this shows that

$$
0 \leq M_{2} L(x)-\operatorname{Var}_{x} L \leq M_{2} D(x), x \in I .
$$

Now define

$$
E(L)(x):=\sum_{j=0}^{\infty}\left(\operatorname{Var} A_{j}\right) p_{j}(x), x \in I
$$

We have

$$
\begin{aligned}
M_{2} L(x) & =L\left(e_{1}-x e_{0}\right)^{2}(x) \\
& =\sum_{j=0}^{\infty}\left(A_{j}\left(e_{2}\right)-2 x b_{j}+x^{2}\right) p_{j}(x) \\
& =\sum_{j=0}^{\infty}\left(A_{j}\left(e_{2}\right)-b_{j}^{2}\right) p_{j}(x)+\sum_{j=0}^{\infty}\left(b_{j}-x\right)^{2} p_{j}(x) .
\end{aligned}
$$

With (4) and (6) this leads to

$$
M_{2} L(x)=E(L)(x)+M_{2} D(x), x \in I .
$$

Combined with (7), this yields

$$
E(L)(x) \leq \operatorname{Var}_{x} L, x \in I .
$$


Finally, let $f \in H \cap C^{2}(I)$ and suppose that $\left\|f^{\prime \prime}\right\|_{\infty}<\infty$. Then by Taylor's formula,

$$
\left|f(t)-f\left(b_{j}\right)-\left(t-b_{j}\right) f^{\prime}\left(b_{j}\right)\right| \leq \frac{1}{2}\left(t-b_{j}\right)^{2}\left\|f^{\prime \prime}\right\|_{\infty}, t \in I .
$$

This entails

$$
\left|A_{j}(f)-f\left(b_{j}\right)\right| \leq \frac{1}{2}\left(\operatorname{Var} A_{j}\right)\left\|f^{\prime \prime}\right\|_{\infty}
$$

Moreover, according to (2) and (5),

$$
|L f-D f| \leq \sum_{j=0}^{\infty}\left|A_{j}(f)-f\left(b_{j}\right)\right| p_{j}
$$

and so

$$
|L f-D f| \leq \frac{1}{2}\left\|f^{\prime \prime}\right\|_{\infty} \sum_{j=0}^{\infty}\left(\operatorname{Var} A_{j}\right) p_{j}
$$

We conclude that

$$
|L f(x)-D f(x)| \leq \frac{1}{2}\left\|f^{\prime \prime}\right\|_{\infty} E(L)(x), x \in I .
$$

Using (12) we see that $E(L)(x)$ shows how far is $L$ from $D$.

\section{Linking operators and discrete operators}

In $[10,11]$ Păltănea introduced operators depending on a parameter $\rho \in \mathbb{R}^{+}$, which constitute a non-trivial link between the Bernstein and Szász-Mirakjan operators, respectively, and their genuine Durrmeyer modifications. In [6] this definition was extended to a non-trivial link between Baskakov type operators and genuine BaskakovDurrmeyer type operators and their $k$ th order Kantorovich modification. For $k=1$ this means a link between the Kantorovich modification of Baskakov type and BaskakovDurrmeyer type operators. In this paper we consider the whole family of linking operators depending on an arbitrary real parameter $c$.

In what follows for $c \in \mathbb{R}$ we use the notations

$$
a^{c, \bar{j}}:=\prod_{l=0}^{j-1}(a+c l), a^{c, \underline{j}}:=\prod_{l=0}^{j-1}(a-c l), j \in \mathbb{N} ; \quad a^{c, \overline{0}}=a^{c, \underline{0}}:=1
$$

In the following definitions of the operators we omit the parameter $c$ in the notations in order to reduce the necessary sub and superscripts. 
Let $c \in \mathbb{R}, n \in \mathbb{R}^{+}, n>c$ for $c \geq 0$ and $-n / c \in \mathbb{N}$ for $c<0$. Furthermore let $\rho \in \mathbb{R}^{+}, j \in \mathbb{N}_{0}, x \in I_{c}$ with $I_{c}=[0, \infty)$ for $c \geq 0$ and $I_{c}=[0,-1 / c]$ for $c<0$. Then the basis functions are given by

$$
p_{n, j}(x)= \begin{cases}\frac{n^{j}}{j !} x^{j} e^{-n x} & , c=0 \\ \frac{n^{c, j}}{j !} x^{j}(1+c x)^{-\left(\frac{n}{c}+j\right)} & , c \neq 0 .\end{cases}
$$

In the following definitions we assume that the function $f: I_{c} \longrightarrow \mathbb{R}$ is given in such a way that the corresponding integrals and series are convergent. The operators of Baskakov-type are defined by

$$
\left(B_{n, \infty} f\right)(x)=\sum_{j=0}^{\infty} p_{n, j}(x) f\left(\frac{j}{n}\right)
$$

and the genuine Baskakov-Durrmeyer type operators are denoted by

$$
\begin{aligned}
\left(B_{n, 1} f\right)(x)= & f(0) p_{n, 0}(x)+f\left(-\frac{1}{c}\right) p_{n,-\frac{n}{c}}(x) \\
& +\sum_{j=1}^{-\frac{n}{c}-1} p_{n, j}(x)(n+c) \int_{0}^{-\frac{1}{c}} p_{n+2 c, j-1}(t) f(t) d t
\end{aligned}
$$

for $c<0$ and by

$$
\left(B_{n, 1} f\right)(x)=f(0) p_{n, 0}(x)+\sum_{j=1}^{\infty} p_{n, j}(x)(n+c) \int_{0}^{\infty} p_{n+2 c, j-1}(t) f(t) d t
$$

for $c \geq 0$.

Depending on a parameter $\rho \in \mathbb{R}^{+}$the linking operators are given by

$$
\left(B_{n, \rho} f\right)(x)=\sum_{j=0}^{\infty} F_{n, j}^{\rho}(f) p_{n, j}(x)
$$

where

$$
F_{n, j}^{\rho}(f)= \begin{cases}f(0) & , j=0, c \in \mathbb{R} \\ f\left(-\frac{1}{c}\right) & , j=-\frac{n}{c}, c<0 \\ \int_{I_{c}} \mu_{n, j}^{\rho}(t) f(t) d t & , \text { otherwise }\end{cases}
$$


with

$$
\mu_{n, j}^{\rho}(t)= \begin{cases}\frac{(-c)^{j \rho}}{B\left(j \rho,-\left(\frac{n}{c}+j\right) \rho\right)} t^{j \rho-1}(1+c t)^{-\left(\frac{n}{c}+j\right) \rho-1} & , c<0, \\ \frac{(n \rho)^{j \rho}}{\Gamma(j \rho)} t^{j \rho-1} e^{-n \rho t} & , c=0, \\ \frac{c^{j \rho}}{B\left(j \rho, \frac{n}{c} \rho+1\right)} t^{j \rho-1}(1+c t)^{-\left(\frac{n}{c}+j\right) \rho-1} & , c>0 .\end{cases}
$$

By $B(x, y)=\int_{0}^{1} t^{x-1}(1-t)^{y-1} d t, x, y>0$ we denote Euler's Beta function and by $\Gamma$ the Gamma function.

Note that in case $c<0$ the sums in (13) and (15) are finite, as $p_{n, j}(x)=0$ for $j>-n / c$. The $k$ th order Kantorovich modification of the operators $B_{n, \rho}$ are defined by

$$
B_{n, \rho}^{(k)}:=D^{k} \circ B_{n, \rho} \circ I_{k}
$$

where $D^{k}$ denotes the $k$ th order ordinary differential operator and

$$
I_{k} f=f, \text { if } k=0, \text { and }\left(I_{k} f\right)(x)=\int_{0}^{x} \frac{(x-t)^{k-1}}{(k-1) !} f(t) d t, \text { if } k \in \mathbb{N}
$$

For $k=0$ we omit the superscript $(k)$ as indicated by the definition above.

We recall some results concerning $\lim _{\rho \rightarrow \infty} B_{n, \rho}^{(k)}$.

In [3, Theorem 2.3] Gonska and Păltănea proved for $c=-1$ the convergence of the operators $B_{n, \rho}$ to the classical Bernstein operator $B_{n, \infty}$, i.e., they proved that for every $f \in C[0,1]$

$$
\lim _{\rho \rightarrow \infty} B_{n, \rho} f=B_{n, \infty} f \text { uniformly on }[0,1] .
$$

From [5] (see the consideration of the special case $\rho \rightarrow \infty$ after Remark 2 there) we know that for each polynomial $q$

$$
\lim _{\rho \rightarrow \infty} B_{n, \rho}^{(k)} q=B_{n, \infty}^{(k)} q
$$

uniformly on $[0,1]$ for $c<0$ and uniformly on every compact subinterval of $[0, \infty)$ for $c \geq 0$.

Let $c=-1$ and $\varepsilon>0$ be arbitrary. As the space of polynomials $\mathcal{P}$ is dense in $L_{p}[0,1],\|\cdot\|_{p}, 1 \leq p<\infty$ and $C[0,1],\|\cdot\|_{\infty}, p=\infty$, we can choose a polynomial $q$, such that $\|f-q\|_{p}<\varepsilon$. Then

$$
\left\|\left(B_{n, \rho}^{(k)}-B_{n, \infty}^{(k)}\right) f\right\|_{p} \leq\left\|B_{n, \rho}^{(k)}(f-q)\right\|_{p}+\left\|B_{n, \infty}^{(k)}(f-q)\right\|_{p}+\left\|\left(B_{n, \rho}^{(k)}-B_{n, \infty}^{(k)}\right) q\right\|_{p} .
$$


As the operators $B_{n, \rho}^{(k)}$ and $B_{n, \infty}^{(k)}$ are bounded (see [5, Corollary 1] and [2, (3)] for the images of $e_{0}$ ) we immediately get

$$
\lim _{\rho \rightarrow \infty}\left\|\left(B_{n, \rho}^{(k)}-B_{n, \infty}^{(k)}\right) f\right\|_{p}=0
$$

for each $f \in L_{p}[0,1],\|\cdot\|_{p}, 1 \leq p<\infty$ and $C[0,1],\|\cdot\|_{\infty}, p=\infty$.

Furthermore, for $c=0$ the following convergence result was proved in [12, Theorem 4].

Let $c=0$. Assume that $f:[0, \infty) \longrightarrow \mathbb{R}$ is integrable and there exist constants $M>0, q \geq 0$ such that $|f(t)| \leq M e^{q t}$ for $t \in[0, \infty)$. Then for any $b>0$ there is $\rho_{0}>0$, such that $B_{n, \rho} f$ exists for all $\rho \geq \rho_{0}$ and we have

$$
\lim _{\rho \rightarrow \infty}\left(B_{n, \rho} f\right)(x)=\left(B_{n, \infty} f\right)(x), \text { uniformly for } x \in[0, b] .
$$

A different function space was considered in [1, Lemma 5, Corollary 3] for the case $c \geq 0$.

Let $f \in C^{2}[0, \infty)$ with $\left\|f^{\prime \prime}\right\|_{\infty}<\infty$. Then we have

$$
\lim _{\rho \rightarrow \infty}\left(B_{n, \rho} f\right)(x)=\left(B_{n, \infty} f\right)(x),
$$

uniformly on every compact subinterval of $[0, \infty)$.

We will use the following representations for the linking operators (see [7, Theorem 2] and [8, Theorem 4] in case $\rho \in \mathbb{N}$.

$c=-1$ : Let $n, k \in \mathbb{N}, n-k \geq 1, \rho \in \mathbb{N}$ and $f \in L_{1}[0,1]$. Then we have the representation

$$
\begin{aligned}
B_{n, \rho}^{(k)}(f ; x)= & \frac{n !(n \rho-1) !}{(n-k) !(n \rho+k-2) !} \sum_{j=0}^{n-k} p_{n-k, j}(x) \\
& \times \int_{0}^{1} \sum_{i_{1}=0}^{\rho-1} \cdots \sum_{i_{k}=0}^{\rho-1} p_{n \rho+k-2, j \rho+i_{1}+\cdots+i_{k}+k-1}(t) f(t) d t .
\end{aligned}
$$

$c \geq 0$ : Let $n, k \in \mathbb{N}, n-k \geq 1, \rho \in \mathbb{N}$ and $f \in W_{n}^{\rho}$ where $W_{n}^{\rho}$ denotes the space of functions $f \in L_{1, l o c}[0, \infty)$ satisfying certain growth conditions, i.e., there exist constants $M>0,0 \leq q<n \rho+c$, such that a.e. on $[0, \infty)$

$$
\begin{aligned}
& |f(t)| \leq M e^{q t} \text { for } c=0 \\
& |f(t)| \leq M t^{\frac{q}{c}} \text { for } c>0
\end{aligned}
$$

Then we have the representation

$$
B_{n, \rho}^{(k)}(f ; x)=\frac{n^{c, \bar{k}}}{(n \rho)^{c, k-1}} \sum_{j=0}^{\infty} p_{n+k c, j}(x)
$$




$$
\times \int_{0}^{\infty} \sum_{i_{1}=0}^{\rho-1} \cdots \sum_{i_{k}=0}^{\rho-1} p_{n \rho-c(k-2), j \rho+i_{1}+\cdots+i_{k}+k-1}(t) f(t) d t
$$

For the images of monomials explicit representations are known from [5, Theorem 2] and [6, Theorem 2]. We will need (see [5, Corollary 1] and [6, Corollary 2] the images of the first monomials.

$$
\begin{aligned}
\left(B_{n, \rho}^{(k)} e_{0}\right)(x)= & \frac{\rho^{k}}{(n \rho)^{c, \underline{k}}} \cdot n^{c, \bar{k}} \\
\left(B_{n, \rho}^{(k)} e_{1}\right)(x)= & \frac{\rho^{k+1}}{(n \rho)^{c, k+1}} \cdot n^{c, \bar{k}}\left[\frac{1}{2} k\left(1+\frac{1}{\rho}\right)+(n+c k) x\right], \\
\left(B_{n, \rho}^{(k)} e_{2}\right)(x)= & \frac{\rho^{k+2}}{(n \rho)^{c, k+2}} \cdot n^{c, \bar{k}}\left[\frac{1}{2} k\left(\frac{3 k+1}{6}+\frac{k+1}{\rho}+\frac{3 k+5}{6 \rho^{2}}\right)\right. \\
& \left.+(n+c k)\left((k+1)\left(1+\frac{1}{\rho}\right) x+(n+c(k+1)) x^{2}\right)\right] .
\end{aligned}
$$

We consider the operators

$$
V_{n, \rho}^{(k)}:=\frac{(n \rho)^{c, \underline{k}}}{\rho^{k} n^{c, \bar{k}}} B_{n, \rho}^{(k)}
$$

for which we have $V_{n, \rho}^{(k)} e_{0}=e_{0}$.

They are of the form (2); more precisely,

$$
V_{n, \rho}^{(k)} f:=\sum_{j=0}^{\infty} A_{n, \rho, j}^{(k)}(f) p_{n+k c, j},
$$

where [see (17), (18) and (20)]

$$
\begin{aligned}
& A_{n, \rho, j}^{(k)}(f) \\
& \quad:=\frac{n \rho-(k-1) c}{\rho^{k}} \sum_{i_{1}=0}^{\rho-1} \cdots \sum_{i_{k}=0}^{\rho-1} \int_{I_{c}} p_{n \rho-(k-2) c, j \rho+i_{1}+\cdots+i_{k}+k-1}(t) f(t) d t .
\end{aligned}
$$

Again in (21) and in the corresponding formulas below the sum is finite in case $c<0$.

If $n \rho>k c$, we can consider the barycenter of $A_{n, \rho, j}^{(k)}$. As $t p_{m+c, l-1}(t)=\frac{l}{m} p_{m, l}(t)$ and $\int_{0}^{\infty} p_{m, l}(t) d t=\frac{1}{m+1}$ we can calculate

$$
b_{n, \rho, j}^{(k)}:=A_{n, \rho, j}^{(k)}\left(e_{1}\right)
$$




$$
\begin{aligned}
& =\frac{n \rho-k c+c}{\rho^{k}} \sum_{i_{1}=0}^{\rho-1} \cdots \sum_{i_{k}=0}^{\rho-1} \int_{I_{c}} p_{n \rho-c(k-2), j \rho+i_{1}+\cdots+i_{k}+k-1}(t) t d t \\
& =\frac{n \rho-k c+c}{\rho^{k}} \sum_{i_{1}=0}^{\rho-1} \cdots \sum_{i_{k}=0}^{\rho-1} \frac{j \rho+i_{1}+\ldots i_{k}+k}{n \rho-c k+c} \cdot \frac{1}{n \rho-c k} \\
& =\frac{1}{\rho^{k}(n \rho-c k)}\left\{\sum_{i_{1}=0}^{\rho-1} \cdots \sum_{i_{k}=0}^{\rho-1}(j \rho+k)+\sum_{i_{1}=0}^{\rho-1} \cdots \sum_{i_{k}=0}^{\rho-1}\left(i_{1}+\ldots i_{k}\right)\right\} \\
& =\frac{1}{\rho^{k}(n \rho-c k)}\left\{(j \rho+k) \rho^{k}+\rho^{k-1} \cdot \frac{(\rho-1) \rho}{2} \cdot k\right\} \\
& =\frac{(2 j+k) \rho+k}{2(n \rho-k c)} .
\end{aligned}
$$

The discrete operators (5) associated with $V_{n, \rho}^{(k)}$ are

$$
D_{n, \rho}^{(k)} f:=\sum_{j=0}^{\infty} f\left(b_{n, \rho, j}^{(k)}\right) p_{n+k c, j} .
$$

Under the form

$$
D_{n, \rho}^{(k)} f=\sum_{j=0}^{\infty} f\left(\frac{j+\frac{(\rho+1) k}{2 \rho}}{(n+k c)-\frac{\rho+1}{\rho} k c}\right) p_{n+k c, j},
$$

we see that $D_{n, \rho}^{(k)}$ is a Stancu-type modification of the operator (13)

$$
B_{n+k c, \infty} f=\sum_{j=0}^{\infty} f\left(\frac{j}{n+k c}\right) p_{n+k c, j} .
$$

A direct calculation similar to (23) shows that

$$
\begin{aligned}
& A_{n, \rho, j}^{(k)}\left(e_{2}\right) \\
& :=\frac{12(j \rho+k)(j \rho+k+1)+4 k[(3 j+1) \rho+3 k+1](\rho-1)+3 k(k-1)(\rho-1)^{2}}{12(n \rho-k c)(n \rho-k c-c)} .
\end{aligned}
$$

Now (4), (23) and (26) imply

$$
\begin{aligned}
& \operatorname{Var} A_{n, \rho, j}^{(k)} \\
& :=\frac{k n \rho^{3}+6[k(2 j c+n)+2 j(j c+n)] \rho^{2}+5 k n \rho+2 k^{2} c\left(\rho^{2}-1\right)}{12(n \rho-k c)^{2}(n \rho-k c-c)} .
\end{aligned}
$$


With the notation $E_{n, \rho}^{(k)}:=E\left(V_{n, \rho}^{(k)}\right)$ and using (8) and (27), we get

$$
\begin{aligned}
& E_{n, \rho}^{(k)}(x) \\
& :=\frac{k n \rho^{3}+[12 x(1+c x)(k c+n)(k c+n+c)+6 k n] \rho^{2}+5 k n \rho+2 k^{2} c\left(\rho^{2}-1\right)}{12(n \rho-k c)^{2}(n \rho-k c-c)} .
\end{aligned}
$$

Here are some particular values:

$$
\begin{aligned}
\operatorname{Var} A_{n, \infty, j}^{(k)} & =\frac{k}{12 n^{2}}, \quad \operatorname{Var} A_{n, 1, j}^{(k)} \\
\text { and } E_{n, \infty}^{(k)}(x) & =\frac{k}{12 n^{2}}, \quad E_{n, 1}^{(k)}(x)=\frac{(j+k)(n+j c)}{(n-k c)^{2}(n-k c-c)} \\
(n-k c)^{2}(n-(k+1) c) & =\frac{x(n+k)(n+(k+1) c)+k n}{}
\end{aligned}
$$

For $c=0$,

$$
\operatorname{Var} A_{n, \rho, j}^{(k)}=\frac{k \rho^{2}+6(k+2 j) \rho+5 k}{12 n^{2} \rho^{2}}, \quad E_{n, \rho}^{(k)}=\frac{12 n \rho x+k(\rho+1)(\rho+5)}{12 n^{2} \rho^{2}}
$$

The images of the first monomials under $V_{n, \rho}^{(k)}$ can be deduced from (19); using them we find

$$
\begin{aligned}
& \operatorname{Var}_{x} V_{n, \rho}^{(k)}:=V_{n, \rho}^{(k)} e_{2}(x)-\left(V_{n, \rho}^{(k)} e_{1}(x)\right)^{2} \\
& =\frac{12 n x(1+c x)(n+k c) \rho^{2}(\rho+1)+2 k(3 n+k c) \rho^{2}+k n \rho\left(\rho^{2}+5\right)-2 k^{2} c}{12(n \rho-k c)^{2}(n \rho-k c-c)} .
\end{aligned}
$$

As explained in Sect. 2, $\operatorname{Var}_{x} V_{n, \rho}^{(k)}$ shows how far is the functional $f \mapsto V_{n, \rho}^{(k)} f(x)$ from the point evaluation at its barycenter $\frac{2(n+k c) \rho x+k(\rho+1)}{2(n \rho-k c)}$ which can be calculated by using (19) and (20).

This assertion, and other similar ones, are made more precise in the following Theorem.

Theorem 1 Let $\left\|f^{\prime \prime}\right\|_{\infty}<\infty$. Then

$$
\begin{aligned}
\left|A_{n, \rho, j}^{(k)}(f)-f\left(\frac{(2 j+k) \rho+k}{2(n \rho-k c)}\right)\right| & \leq \frac{1}{2}\left\|f^{\prime \prime}\right\|_{\infty} \operatorname{Var} A_{n, \rho, j}^{(k)}, \\
\left|V_{n, \rho}^{(k)} f(x)-D_{n, \rho}^{(k)} f(x)\right| & \leq \frac{1}{2} E_{n, \rho}^{(k)}(x)\left\|f^{\prime \prime}\right\|_{\infty} \\
\left|V_{n, \rho}^{(k)} f(x)-f\left(\frac{2(n+k c) \rho x+k(\rho+1)}{2(n \rho-k c)}\right)\right| & \leq \frac{1}{2}\left\|f^{\prime \prime}\right\|_{\infty} \operatorname{Var}_{x} V_{n, \rho}^{(k)} .
\end{aligned}
$$

Proof It suffices to apply (11) and (12) to the functionals $A_{n, \rho, j}^{(k)}$ and $f \mapsto V_{n, \rho}^{(k)} f(x)$, respectively to the operators $V_{n, \rho}^{(k)}$.

The case $\rho \rightarrow \infty$ deserves a special attention. 


\section{Theorem 2}

(i) If $\left\|f^{\prime \prime}\right\|_{\infty}<\infty$, then

$$
\left\|V_{n, \infty}^{(k)} f-D_{n, \infty}^{(k)} f\right\|_{\infty} \leq \frac{k}{24 n^{2}}\left\|f^{\prime \prime}\right\|_{\infty}
$$

(ii) For an arbitrary $f$ in the domain of $V_{n, \infty}^{(k)}$,

$$
\left\|V_{n, \infty}^{(k)} f-D_{n, \infty}^{(k)} f\right\|_{\infty} \leq \omega\left(f ; \frac{k}{2 n}\right) .
$$

Proof Equation (36) follows from (35) and (30). In order to prove (37), let us remark that

$$
D_{n, \infty}^{(k)} f(x)=\sum_{j=0}^{\infty} f\left(\frac{2 j+k}{2 n}\right) p_{n+k c, j}(x)
$$

and

$$
\begin{aligned}
V_{n, \infty}^{(k)} f(x) & =\frac{n^{k}}{n^{c, k}} B_{n, \infty}^{(k)} f(x) \\
& =n^{k} \sum_{j=0}^{\infty} p_{n+k c, j}(x) \Delta_{\frac{1}{n}}^{k}\left(I_{k} f\right)\left(\frac{j}{n}\right) \\
& =k ! \sum_{j=0}^{\infty}\left[\frac{j}{n}, \frac{j+1}{n}, \ldots, \frac{j+k}{n} ; I_{k} f\right] p_{n+k c, j}(x) .
\end{aligned}
$$

According to the mean value theorem for divided differences there exists $x_{n, k, j} \in$ $\left[\frac{j}{n}, \frac{j+k}{n}\right]$ such that

$$
k !\left[\frac{j}{n}, \frac{j+1}{n}, \ldots, \frac{j+k}{n} ; I_{k} f\right]=f\left(x_{n, k, j}\right) .
$$

Consequently,

$$
V_{n, \infty}^{(k)} f(x)=\sum_{j=0}^{\infty} f\left(x_{n, k, j}\right) p_{n+k c, j}(x)
$$

Since $x_{n, k, j} \in\left[\frac{j}{n}, \frac{j+k}{n}\right]$, it follows that $\left|x_{n, k, j}-\frac{2 j+k}{2 n}\right| \leq \frac{k}{2 n}$, and so

$$
\left|\left(V_{n, \infty}^{(k)} f-D_{n, \infty}^{(k)} f\right)(x)\right| \leq \sum_{j=0}^{\infty}\left|f\left(x_{n, k, j}\right)-f\left(\frac{2 j+k}{2 n}\right)\right| p_{n+k c, j}(x)
$$




$$
\leq \omega\left(f ; \frac{k}{2 n}\right), \text { for all } x \geq 0
$$

This proves (37).

In the following Theorem we give an estimate for the difference of $V_{n, \rho}^{(k)} f(x)$ and $V_{n, \infty}^{(k)} f(x)$.

Theorem 3 If $\left\|f^{\prime}\right\|_{\infty}<\infty$ and $\left\|f^{\prime \prime}\right\|_{\infty}<\infty$, then

$$
\begin{aligned}
& \left|V_{n, \rho}^{(k)} f(x)-V_{n, \infty}^{(k)} f(x)\right| \\
& \quad \leq \frac{1}{2}\left\|f^{\prime \prime}\right\|_{\infty}\left[E_{n, \rho}^{(k)}(x)+E_{n, \infty}^{(k)}(x)\right]+\left\|f^{\prime}\right\|_{\infty} \cdot \frac{k(n+k c)(1+c x)}{2 n(n \rho-k c)}
\end{aligned}
$$

with $E_{n, \rho}^{(k)}(x)$ and $E_{n, \infty}^{(k)}(x)$ given in (28) and (30).

Proof By using (34) we derive

$$
\begin{aligned}
& \left|V_{n, \rho}^{(k)} f(x)-V_{n, \infty}^{(k)} f(x)\right| \\
& \leq\left|V_{n, \rho}^{(k)} f(x)-D_{n, \rho}^{(k)} f(x)\right|+\left|V_{n, \infty}^{(k)} f(x)-D_{n, \infty}^{(k)} f(x)\right| \\
& \quad+\left|D_{n, \rho}^{(k)} f(x)-D_{n, \infty}^{(k)} f(x)\right| \\
& \leq \frac{1}{2}\left\|f^{\prime \prime}\right\|_{\infty}\left[E_{n, \rho}^{(k)}(x)+E_{n, \infty}^{(k)}(x)\right]+\left|D_{n, \rho}^{(k)} f(x)-D_{n, \infty}^{(k)} f(x)\right| .
\end{aligned}
$$

Application of the mean value theorem leads to

$$
\begin{aligned}
& \left|D_{n, \rho}^{(k)} f(x)-D_{n, \infty}^{(k)} f(x)\right| \\
& \quad \leq \sum j=0^{\infty} p_{n+c k, j}(x)\left|f\left(\frac{(2 j+k) \rho+k}{2(n \rho-k c}\right)-f\left(\frac{2 j+k}{2 n}\right)\right| \\
& \quad \leq\left\|f^{\prime}\right\|_{\infty} \sum j=0^{\infty} p_{n+c k, j}(x) \frac{k n+(2 j+k) k c}{2 n(n \rho-k c} \\
& \quad=\left\|f^{\prime}\right\|_{\infty} \frac{k(n+k c)(1+c x)}{2 n(n \rho-k c)} .
\end{aligned}
$$

Together with (40) we get our proposition.

With the definition of the operators $V_{n, \rho}^{(k)}$ we get from Theorem 3

\section{Corollary 1}

$$
\begin{aligned}
& \left|B_{n, \rho}^{(k)} f(x)-B_{n, \infty}^{(k)} f(x)\right| \\
& \quad \leq \frac{\rho^{k} n^{c, \bar{k}}}{(n \rho)^{c, \underline{k}}}\left\{\frac{1}{2}\left\|f^{\prime \prime}\right\|_{\infty}\left[E_{n, \rho}^{(k)}(x)+E_{n, \infty}^{(k)}(x)\right]+\left\|f^{\prime}\right\|_{\infty} \cdot \frac{k(n+k c)(1+c x)}{2 n(n \rho-k c)}\right\}
\end{aligned}
$$


Let us return to $\operatorname{Var} A_{n, \rho, j}^{(k)}, E_{n, \rho}^{(k)}$ and $\operatorname{Var}_{x} V_{n, \rho}^{(k)}$. Their roles were illustrated in Theorem 1; some properties of them are presented in the following result.

Theorem $4 \operatorname{Var} A_{n, \rho, j}^{(k)}, E_{n, \rho}^{(k)}$ and $\operatorname{Var}_{x} V_{n, \rho}^{(k)}$ are:

(a) increasing with respect to $k$,

(b) increasing with respect to $c$,

(c) decreasing with respect to $\rho$.

Proof (a) and (b) follow easily from (27), (28) and (33).

Now let us denote $a:=\frac{k c}{n}$ and $b:=\frac{k c+c}{n}$. Then (27) can be rewritten as

$$
\begin{aligned}
\operatorname{Var} A_{n, \rho, j}^{(k)}= & \frac{1}{6 n^{3}}\left\{3[k(2 j c+n)+2 j(j c+n)]+k^{2} c\right\}\left(\frac{\rho}{\rho-a}\right)^{2} \frac{1}{\rho-b} \\
& +\frac{k}{12 n^{2}}\left\{\frac{\rho^{2}+3}{(\rho-a)^{2}} \frac{\rho}{\rho-b}+\frac{2}{\rho-a} \frac{1}{\rho-b}\right\},
\end{aligned}
$$

and this shows that $\operatorname{Var} A_{n, \rho, j}^{(k)}$ is decreasing w.r.t. $\rho$.

A similar transformation of (28) [or an inspection of (8)] reveals that $E_{n, \rho}^{(k)}$ is also decreasing w.r.t. $\rho$.

Finally (33) can be put under the form

$$
\begin{aligned}
\operatorname{Var}_{x} V_{n, \rho}^{(k)}= & \frac{x(1+c x)(n+k c)}{n^{2}}\left(\frac{\rho}{\rho-a}\right)^{2} \frac{\rho+1}{\rho-b}+\frac{k c+3 n}{6 n^{3}}\left(\frac{\rho}{\rho-a}\right)^{2} \frac{1}{\rho-b} \\
& +\frac{k}{12 n^{2}}\left\{\frac{\rho^{2}+3}{(\rho-a)^{2}} \frac{\rho}{\rho-b}+\frac{2}{\rho-a} \frac{1}{\rho-b}\right\},
\end{aligned}
$$

which shows that $\operatorname{Var}_{x} V_{n, \rho}^{(k)}$ is decreasing w.r.t. $\rho$.

\section{Some complementary results}

The starting point for this article was the following remark.

Let $n \in \mathbb{N}, c=-1, x \in[0,1], 1 \leq k<n, f \in C[0,1]$. Then (39) takes the form

$$
V_{n, \infty}^{(k)} f(x)=\sum_{j=0}^{n-k} f\left(x_{n, k, j}\right) p_{n-k, j}(x),
$$

for suitable $x_{n, k, j} \in\left[\frac{j}{n}, \frac{j+k}{n}\right]$. In (41) $p_{n-k, j}(x)$ are the very classical Bernstein fundamental polynomials, i.e.,

$$
p_{n-k, j}(x):=\left(\begin{array}{c}
n-k \\
j
\end{array}\right) x^{j}(1-x)^{n-k-j}, x \in[0,1] .
$$


The classical Bernstein polynomials are, of course,

$$
B_{n-k} f(x):=\sum_{j=0}^{n-k} f\left(\frac{j}{n-k}\right) p_{n-k, j}(x)
$$

Both $x_{n, k, j}$ and $\frac{j}{n-k}$ are in $\left[\frac{j}{n}, \frac{j+k}{n}\right]$, so that $\left|x_{n, k, j}-\frac{j}{n-k}\right| \leq \frac{k}{n}$. Now from (41) and (42) we get

$$
\left\|V_{n, \infty}^{(k)}-B_{n-k} f\right\|_{\infty} \leq \omega\left(f ; \frac{k}{n}\right)
$$

In particular, $V_{n, \infty}^{(1)}$ is the classical Kantorovich operator $K_{n-1}$ on $C[0,1]$. So (43) implies

$$
\left\|K_{n-1} f-B_{n-1} f\right\|_{\infty} \leq \omega\left(f ; \frac{1}{n}\right), f \in C[0,1] .
$$

Let us return to an arbitrary $c$ and to

$$
B_{n, \infty} f(x):=\sum_{j=0}^{\infty} p_{n, j}(x) f\left(\frac{j}{n}\right) .
$$

The sum $S_{n}(x)=S_{n, c}(x):=\sum_{j=0}^{\infty} p_{n, j}^{2}(x)$ was investigated in a series of papers, see $[14,15]$ and the references given there.

It is known that it is logarithmically convex (see [15]) and

$$
\begin{aligned}
& S_{n, c}(x)=\frac{1}{\pi} \int_{0}^{1}\left(t+(1-t)(1+2 c x)^{2}\right)^{-\frac{n}{c}} \frac{d t}{\sqrt{t(1-t)}}, c \neq 0, \\
& S_{n, 0}(x)=\frac{1}{\pi} \int_{-1}^{1} e^{-2 n x(1+t)} \frac{d t}{\sqrt{1-t^{2}}}, \\
& S_{n, c}(x) \leq(4(n+c) x(1+c x)+1)^{-\frac{n}{2(n+c)}} .
\end{aligned}
$$

Moreover (see [4,14]),

$$
\left|B_{n, \infty}(f g)(x)-B_{n, \infty} f(x) B_{n, \infty} g(x)\right| \leq \frac{1}{2}\left(1-S_{n, c}(x)\right) \operatorname{osc}_{n}(f) \operatorname{osc}_{n}(g),
$$

where $\operatorname{osc}_{n}(f):=\sup \left\{\left|f\left(\frac{j}{n}\right)-f\left(\frac{i}{n}\right)\right|: i, j \in \mathbb{N}_{0}\right\}$.

Let $n$ and $x$ be fixed; consider the functional $f \mapsto B_{n, \infty} f(x)$. Then, roughly speaking $1-S_{n, c}(x)$ shows how far is the functional from being a multiplicative functional, and ultimately how far is it from being a point evaluation.

Consider $\left(p_{n, 0}(x), p_{n, 1}(x), \ldots\right)$ as a probability distribution parameterized by $x$. Then $1-S_{n, c}(x)$ is the associated Tsallis entropy. Moreover, $S_{n}(x)$ is viewed as one of the indices measuring the inequality and diversity, i.e., the degree of uniformness of the distribution (see [9, pp. 556-559]). 


\section{Compliance with ethical standards}

Conflict of interest The authors declare that they have no competing interests.

Open Access This article is licensed under a Creative Commons Attribution 4.0 International License, which permits use, sharing, adaptation, distribution and reproduction in any medium or format, as long as you give appropriate credit to the original author(s) and the source, provide a link to the Creative Commons licence, and indicate if changes were made. The images or other third party material in this article are included in the article's Creative Commons licence, unless indicated otherwise in a credit line to the material. If material is not included in the article's Creative Commons licence and your intended use is not permitted by statutory regulation or exceeds the permitted use, you will need to obtain permission directly from the copyright holder. To view a copy of this licence, visit http://creativecommons.org/licenses/by/4.0/.

\section{References}

1. Baumann, K., Heilmann, M., Raşa, I.: Further results for $k$ th order Kantorovich modification of linking Baskakov type operators. Results Math. 69(3), 297-315 (2016)

2. Gonska, H., Heilmann, M., Raşa, I.: Kantorovich operators of order $k$. Numer. Funct. Anal. Optim. 32, 717-738 (2011)

3. Gonska, H., Păltănea, R.: Simultaneous approximation by a class of Bernstein-Durrmeyer operators preserving linear functions. Czechoslov. Math. J. 60(135), 783-799 (2010)

4. Gonska, H., Raşa, I., Rusu, M.: Chebyshev-Grüss-type inequalities via discrete oscillations. Bul. Acad. Stiinte Repub. Mold. Mat. 1(74), 63-89 (2014)

5. Heilmann, M., Raşa, I.: $k$-th order Kantorovich type modification of the operators $U_{n}^{\rho}$. J. Appl. Funct. Anal. 9(3-4), 320-334 (2014)

6. Heilmann, M., Raşa, I.: $k$-th order Kantorovich modification of linking Baskakov type operators. In: Agrawal, P.N. et al. (ed.) Recent Trends in Mathematical Analysis and its Applications, Proceedings of the Conference ICRTMAA 2014, Rorkee, India, December 2014. Proc. Math. Stat. 143, 229-242 (2015)

7. Heilmann, M., Raşa, I.: A nice representation for a link between Bernstein-Durrmeyer and Kantorovich operators. In: Giri, D. et al. (ed.) Proceedings of ICMC 2017, Haldia, India, January 2017. Springer Proceedings in Mathematics and Computing, pp. 312-320 (2017)

8. Heilmann, M., Raşa, I.: A nice representation for a link between Baskakov- and Szász-MirakjanDurrmeyer operators and their Kantorovich variants. Results Math. 74, 9 (2019). https://doi.org/10. 1007/s00025-018-0932-4

9. Marshall, A.W., Olkin, I., Arnold, B.C.: Inequalities: Theory of Majorization and Its Applications. Springer Series in Statistics, 2nd edn. Springer, New York (2011)

10. Păltănea, R.: A class of Durrmeyer type operators preserving linear functions. Ann. Tiberiu Popoviciu Semin. Funct. Eq. Approx. Conv. 5, 109-117 (2007)

11. Păltănea, R.: Modified Szász-Mirakjan operators of integral form. Carpathian J. Math. 24(3), 378-385 (2008)

12. Păltănea, R.: Simultaneous approximation by a class of Szász-Mirakjan operators. J. Appl. Funct. Anal. 9(3-4), 356-368 (2014)

13. Raşa, I.: Discrete operators associated with certain integral operators. Stud. Univ. Babes-Bolyai Math. 56(2), 537-544 (2011)

14. Raşa, I.: Entropies and Heun functions associated with positive linear operators. Appl. Math. Comput. 268, 422-431 (2015)

15. Raşa, I.: Convexity properties of some entropies. Results Math. 73, 105 (2018)

Publisher's Note Springer Nature remains neutral with regard to jurisdictional claims in published maps and institutional affiliations. 